IASSNS-HEP-97/124

CALT-68-2143

\title{
Singular Monopoles and Supersymmetric Gauge Theories in Three Dimensions
}

\author{
Sergey A. Cherkis* \\ California Institute of Technology \\ Pasadena, CA 91125 \\ Anton Kapustin ${ }^{\dagger}$ \\ School of Natural Sciences, Institute for Advanced Study \\ Olden Lane, Princeton, NJ 08540
}

\begin{abstract}
According to the proposal of Hanany and Witten, Coulomb branches of $N=4 S U(n)$ gauge theories in three dimensions are isometric to moduli spaces of BPS monopoles. We generalize this proposal to gauge theories with matter, which allows us to describe the metrics on their spaces of vacua by means of the hyperkähler quotient construction. To check the identification of moduli spaces a comparison is made with field theory predictions. For $S U(2)$ theory with $k$ fundamental hypermultiplets the Coulomb branch is expected to be the $D_{k}$ ALF gravitational instanton, so our results lead to a construction of such spaces. In the special case of $S U(2)$ theory with four or fewer fundamental hypermultiplets we calculate the complex structures on the moduli spaces and compare them with field-theoretical results. We also discuss some puzzles with brane realizations of threedimensional $N=4$ theories.
\end{abstract}

*Research supported in part by DOE grant DE-FG03-92-ER40701

${ }^{\dagger}$ Research supported in part by DOE grant DE-FG02-90-ER40542 


\section{Introduction}

Realizing supersymmetric gauge theories as theories on D-branes proved to be very useful for identifying their excitations and spaces of vacua. In certain cases this approach allows one to show that the Coulomb branch of the space of vacua is the same as the moduli space of some self-dual Yang-Mills configurations in an auxiliary gauge theory. For example, as described in Ref. [1], the Coulomb branch of $N=4$ supersymmetric $S U(n)$ Yang-Mills theory in three dimensions is the (centered) moduli space of $n S U(2)$ monopoles. There are powerful mathematical methods, such as twistor methods and the ADHM-Nahm construction, developed to describe solutions of the self-duality equation. Using these methods one can compute the metric on the space of vacua. In addition, realization of the same theory by different D-brane configurations clarifies the connection between different mathematical constructions and yields nontrivial predictions about the geometry of the space of vacua.

In this paper we generalize the correspondence between the Coulomb branches and monopole moduli spaces to the case of $D=3, N=4 S U(n)$ gauge theory with $k$ hypermultiplets in the fundamental representation. We show that the Coulomb branch is given by the (centered) moduli space of $U(2)$ "monopoles" on the $A_{k-1}$ ALF space (also called multi-Taub-NUT), as described in Section 2. These "monopoles" turn out to be equivalent to solutions of $U(2)$ Bogomolny equations on $\mathbf{R}^{3}$ with $k$ singularities (Section 3). They can be described by the spectral data in the twistor description [2, 3] or by solutions of Nahm equations.

In the strong coupling limit (i.e. $g \rightarrow \infty$ ) the metric on the Coulomb branch of the $S U(n)$ gauge theory with $k$ hypermultiplets is identical to the metric on the Higgs branch of the mirror gauge theory described in Ref. [1]. The latter does not receive any quantum corrections and can be easily computed. However, going from $g=\infty$ to finite $g$ is highly nontrivial in general. For example, the Coulomb branch of the $S U(2)$ gauge theory with $k$ fundamentals is expected to be the ALF gravitational instanton of type $D_{k}$ 汭, [6], while the $g \rightarrow \infty$ limit is the ALE gravitational instanton. Self-dual ALE metrics have been completely classified by Kronheimer [7], but very little is known about the ALF case (see though Ref. [8]). The monopole methods allow us to construct $D_{k}$ ALF metrics as (infinite-dimensional) hyperkähler quotients.

The brane configurations which we use are the configurations described in 
Ref. [1]; they contain both D and NS5-branes. If one moves D-branes around, naively there seem to be phase transitions in the worldvolume gauge theory, with new matter multiplets appearing when D5 and NS5-branes cross. In Ref. 国 it was argued that phase transitions are actually absent. In some cases this can be explained by creation of a D3-brane when a D5 and an NS5 cross. However there are situations when brane creation does not help and even complicates the picture. In Section 1 we analyze such situations in terms of monopole moduli spaces and argue that phase transitions are still absent. For this to work, certain states of fundamental strings stretched between D-branes must be absent, although simply drawing the picture suggests otherwise.

In Section 5 we construct the moduli spaces of singular monopoles by means of Nahm equations. We work out in detail the examples corresponding to $S U(2)$ gauge theory with up to four fundamental hypermultiplets in Section 6. In Section 7 we present the complex structures on these spaces and compare them with the Seiberg-Witten solutions of the corresponding four-dimensional theories.

In the Appendix we discuss the properties of solutions of self-duality equations on $\mathbf{R}^{3} \times \mathbf{S}^{1}$ and on $A_{k-1}$ ALF space needed for our discussion of phase transitions in Section $\mathbb{6}$.

\section{Brane Configurations}

Following Ref. [1] we consider configurations of D3, D5 and NS5-branes in IIB string theory which leave eight supersymmetries unbroken. Let two parallel NS5-branes be some distance $d$ apart in the $x^{6}$ direction with worldvolumes parallel to $x^{0}, x^{1}, x^{2}, x^{3}, x^{4}, x^{5}$. Let $n$ D3-branes stretch between them in the

$x^{6}$ direction, with other directions of D3's being parallel to $x^{0}, x^{1}, x^{2}$. This configuration of branes is illustrated in Figure 1(a). The theory on D3-branes reduces to the $D=3, N=4 U(n)$ Yang-Mills theory in the infrared limit. Every such configuration of branes corresponds to a particular vacuum of the Yang-Mills theory. As described in Ref. [1], D3-branes look like $S U(2)$ monopoles in the $x^{3}, x^{4}, x^{5}$ directions in the theory on the NS5-branes. Vacua of the $U(n)$ Yang-Mills theory on D3-branes are in one-to-one correspondence with charge $n$ monopoles on the NS5-branes. In order to describe the $S U(n)$ Yang-Mills theory we should fix the center of mass of the D3-branes. Thus vacua of this theory are given by "centered" monopoles. 




(a)

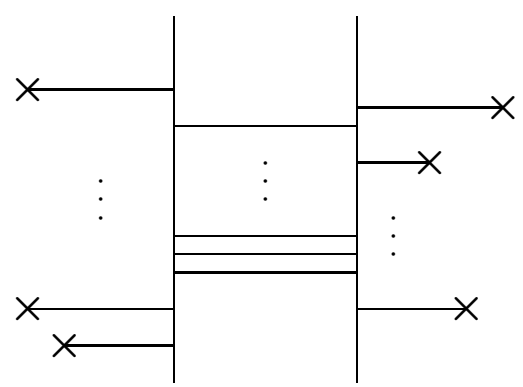

(b)

Figure 1: (a) Parallel NS5-branes (vertical lines) with D3-branes (horizontal lines) suspended between them. The horizontal direction corresponds to $x^{6}$, while the vertical direction corresponds to $x^{3}, x^{4}$, and $x^{5}$ collectively. (b) The same, with additional D5-branes (crosses) connected by D3-branes to NS5-branes.

Now let us add $k$ D5-branes stretching along $x^{0}, x^{1}, x^{2}, x^{7}, x^{8}, x^{9}$ and positioned outside the NS5-branes at points $\vec{p}_{\alpha}$ in the $\left(x^{3}, x^{4}, x^{5}\right)$ plane (see Figure 1(b)). Let each D5-brane be connected by one D3-brane to an NS5brane closest to it. We will call these D3-branes external, to distinguish them from those connecting the two NS5-branes, which we will call internal. From the point of view of the internal D3-branes the low-energy theory is a $U(n)$ gauge theory with $k$ matter hypermultiplets in the fundamental representation. Matter comes from the fundamental strings connecting the internal and external D3-branes. The question is what this configuration looks like in the $S U(2)$ theory on the NS5-branes.

To answer the question we perform $\mathrm{S}$ and $\mathrm{T}$ duality transformations. First we go to the S dual picture thus exchanging D5 and NS5-branes. Then we T dualize along the $x^{6}$ direction (after making it periodic) thus turning IIB string theory into IIA string theory, D5-branes into D6-branes, and NS5branes into an $A_{k-1}$ type ALF space. Tracing the dualities we have the $A_{k-1}$ ALF metric in $\left(x^{3}, x^{4}, x^{5}, \hat{x}^{6}\right)$, with $\hat{x}^{6}$ being the compact direction. Four of the directions of the D6-branes are wrapped on this space.

What do the D3-branes turn into after the dualities? If any of the D3branes were wrapped around $x^{6}$, it would turn into a D2-brane located at a point on the $A_{k-1}$ ALF space. As explained in Ref. [9], this D2-brane would 
look like an instanton in the $U(2)$ theory on the D6-branes. To be more precise, it would be a self-dual $U(2)$ gauge connection on the $A_{k-1}$ ALF space, somewhat resembling Nahm's calorons [10. Note that the $U(2)$ gauge group is broken down to $U(1) \times U(1)$ by a nontrivial Wilson line at infinity (in the original picture this corresponds to a nonzero distance between the NS5-branes.) Therefore there may be states in the theory carrying nonzero magnetic charge. The instanton does not have magnetic charge, and neither does the D3-brane wrapped around the $x^{6}$ direction in the T-dual description. On the other hand, the internal D3-brane does have a magnetic charge, and therefore corresponds to the monopole solution, by which we mean a self-dual connection carrying magnetic charge.

If there were no D5-branes in the original brane configuration, we would be dealing with self-dual connections on $\mathbf{R}^{3} \times \mathbf{S}^{1}$ rather than on the $A_{k-1}$ ALF space. Then the internal D3-branes would correspond to t'Hooft-Polyakov monopoles, i.e. they would not depend on the circle coordinate [11]. It is highly plausible that this remains true when D5 branes are present. Indeed, the well-known maxim "Winding is momentum" implies in this case that in the IIA picture nothing depends on the $\hat{x}^{6}$ direction, since nothing is wound around the T-dual direction in the IIB picture. In the next section we confirm this by exhibiting monopole solutions on the $A_{k-1}$ ALF space which do not depend on the circle coordinate.

We still have not discussed the fortune of the external D3-branes after T-duality. We postpone the answer until the end of the next section.

\section{Monopoles on $A_{k-1}$ ALF Space}

Let us describe precisely what we mean by a monopole on the $A_{k-1}$ ALF space. In coordinates $x^{1}, x^{2}, x^{3}, \theta$ the $A_{k-1}$ ALF metric is

$$
d s^{2}=V d x^{i} d x^{j} \delta_{i j}+V^{-1}\left(d \theta+\omega^{i} d x^{i}\right)^{2}
$$

where $\theta$ has period $4 \pi$ and is T-dual to $x^{6}$, and

$$
V=1+\sum_{\alpha=1}^{k} \frac{1}{\left|\vec{x}-\vec{p}_{\alpha}\right|}, \quad \operatorname{grad} V=\operatorname{curl} \vec{\omega} .
$$

Note that $\omega$ is not a globally defined 1-form; rather it is a connection on a nontrivial $U(1)$ bundle and can only be defined patchwise. 
A $U(2)$ monopole on this space is a smooth self-dual connection $\hat{\mathbf{A}}=$ $\hat{A}_{0} d \theta+\hat{A}_{j} d x^{j}$ on a $U(2)$ vector bundle with a nontrivial holonomy (Wilson line) at infinity and nonzero magnetic charge, whose field strength is independent of $\theta$ in some local gauge. That is, away from the centers $\vec{x}=\vec{p}_{\alpha}$ there is a local gauge transformation $g(\vec{x}, \theta)$, such that $\tilde{A}_{0}=g^{-1} \hat{A}_{0} g-i g^{-1} \partial_{\theta} g$ and $\tilde{A}_{j}=g^{-1} \hat{A}_{j} g-i g^{-1} \partial_{j} g$ are independent of $\theta$.

As $\hat{\mathbf{A}}$ is smooth and the norm of $\frac{\partial}{\partial \theta}$ vanishes when $\vec{x} \rightarrow \vec{p}_{\alpha}$, one necessarily has $\hat{A}_{0}\left(\vec{p}_{\alpha}\right)=0 . g(\vec{x}, \theta)$ approaches a circle action with integer weights $\ell_{\alpha}, \ell_{\alpha}^{\prime}$ near the centers $\vec{p}_{\alpha}$. Then we easily see that after the above-mentioned gauge transformation the eigenvalues of $\tilde{A}_{0}$ approach $\ell_{\alpha} / 2, \ell_{\alpha}^{\prime} / 2$ as $\vec{x} \rightarrow \vec{p}_{\alpha}$.

Since in the new gauge $\mathbf{A}$ does not depend on $\theta$, one may define new fields on $\mathbf{R}^{3}$

$$
\Phi=V \tilde{A}_{0}, \quad A_{i}=\tilde{A}_{i}-\omega_{i} \tilde{A}_{0} .
$$

Kronheimer noticed [2] that these fields satisfy the Bogomolny equation if and only if the initial $\mathbf{A}$ is a self-dual connection on the ALF space. Here $\Phi$ is the Higgs field and $A_{i}$ is the gauge potential on $\mathbf{R}^{3}$. From Eq. (2) it is easy to see that $\Phi$ has singularities at $\vec{x}=\vec{p}_{\alpha}$. Thus monopoles on $A_{k-1}$ ALF space are in one-to-one correspondence with monopoles on $\mathbf{R}^{3}$ with singular Higgs field

$$
\Phi \rightarrow \frac{1}{2\left|\vec{x}-\vec{p}_{\alpha}\right|} \operatorname{diag}\left(\ell_{\alpha}, \ell_{\alpha}^{\prime}\right)
$$

near $\vec{x}=\vec{p}_{\alpha}$ in some gauge. The asymptotic behavior at infinity is the same as for ordinary monopoles,

$$
\Phi \rightarrow \operatorname{diag}\left(1-\frac{n-\sum \ell_{\alpha}}{2 r},-1+\frac{n+\sum \ell_{\alpha}^{\prime}}{2 r}\right) .
$$

We will call such solutions singular U(2) monopoles.

What is the meaning of $n$ in Eq. (5)? The rank $2 U(2)$ bundle decomposes into the sum of eigenspaces of $\Phi, E=M \oplus M^{\prime}$, where $M$ and $M^{\prime}$ are line bundles. It follows from Eq. (4i) and Bogomolny equations that upon restriction to a small sphere around $\vec{x}=\vec{p}_{\alpha}$ the degrees of $M$ and $M^{\prime}$ are $-\ell_{\alpha}$ and $-\ell_{\alpha}^{\prime}$. In other words, there is a point-like Dirac monopole with charges $-\ell_{\alpha},-\ell_{\alpha}^{\prime}$ at $\vec{x}=\vec{p}_{\alpha}$ embedded in the diagonal subgroup of $U(2)$. Similarly, the total magnetic charges of the configuration (the degrees of $M$ and $M^{\prime}$ restricted to a very large sphere) are $n-\sum \ell_{\alpha}$ and $-n-\sum \ell_{\alpha}$. Let us now 


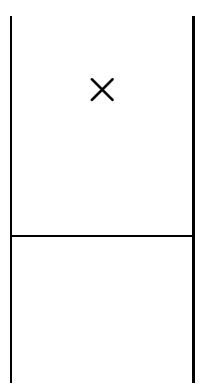

(a)

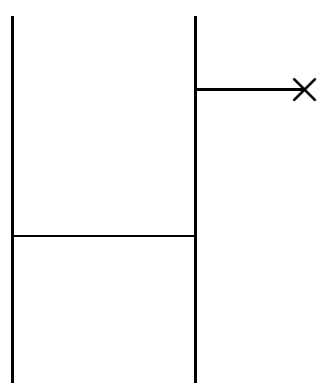

(b)

Figure 2: Starting with configuration (a) and moving the D5-brane to the right one gets configuration (b). In both configurations the low-energy gauge theory on the D3-brane has one hypermultiplet.

focus on the $S U(2)$ subgroup of $U(2)$. Then the total magnetic charge in the $S U(2)$ is $n-\sum\left(\ell_{\alpha}-\ell_{\alpha}^{\prime}\right) / 2$, while the charge carried by the $\alpha$ th Dirac monopole is $-\left(\ell_{\alpha}-\ell_{\alpha}^{\prime}\right) / 2$. Therefore $n$ is naturally interpreted as the number of smooth nonabelian monopoles in the configuration. (Kronheimer [2] calls it nonabelian charge.) One expects that $n \geq 0$, and it can be shown [2] that this is indeed the case.

The Dirac monopole with $\ell=1, \ell^{\prime}=0$ is in fact the reincarnation of the right external D3-brane in the initial brane configuration. This becomes quite obvious if one recalls that the NS5-branes in Figure 1(b) correspond to the two $U(1)$ factors in the diagonal subgroup of $U(2)$, and that the end of the D3 brane ending on the NS5-brane from the left (right) carries magnetic charge $+1(-1)$ in the corresponding $U(1)$. Similarly, the left external D3brane maps under $T$-duality to a Dirac monopole with $\ell=0, \ell^{\prime}=-1$.

To summarize, the configuration of $n$ internal and $k$ external D3-branes corresponds to a solution of $U(2)$ Bogomolny equations with nonabelian charge $n$ and with Higgs field having $k$ singularities as in Eq. (四). Depending on whether the $\alpha$ th D3-brane is right or left we have $\ell_{\alpha}=1, \ell_{\alpha}^{\prime}=0$ or $\ell_{\alpha}=0, \ell_{\alpha}^{\prime}=-1$. 


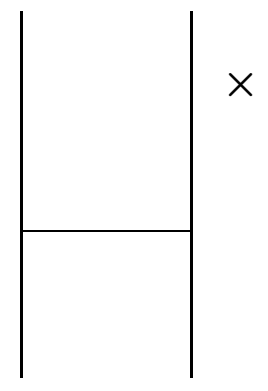

(a)

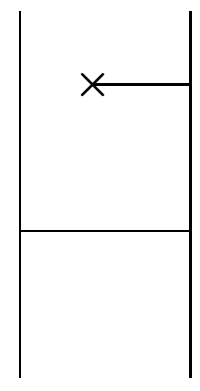

(b)

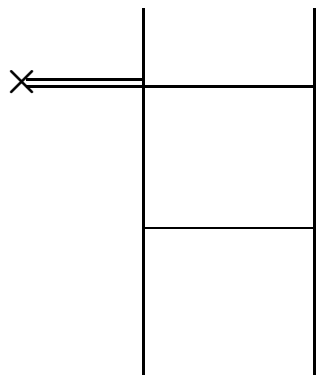

(c)

Figure 3: Starting with configuration (a) and moving the D5-brane to the left one gets configurations (b) and (c).

\section{Phase Transitions?}

The brane configurations in Figures 2(a) and 3(a) correspond to $U(1)$ gauge theories with one and no charged hypermultiplets, respectively. It was noted in Ref. [1] that the position of the D5-brane in the $x^{6}$ direction does not appear as a parameter in the gauge theory. Therefore one could think that there is a phase transition in the gauge theory when D5 and NS5-branes cross. In fact, as explained in Ref. [1], there is no phase transition because a D3-brane is created when D5 crosses NS5. In the case of the configuration in Figure 2(a), moving D5-brane to the right creates a configuration in Figure 2(b). The latter corresponds to a $U(1)$ gauge theory with one hypermultiplet, as there are fundamental strings connecting the external and internal D3-branes.

The picture with singular monopoles described in Section 3 refers to Figure 2(b) on page 6. Figure 2(a), however, presents a puzzle: there are no D3-branes connecting D5 with NS5, so the reasoning of Section 3 seems to imply that the configuration corresponds to a $U(2)$ monopole on the $A_{0}$ ALF space (i.e. the Taub-NUT space) with $n=1, \ell=\ell^{\prime}=0$. It was explained in the previous section that such a monopole is equivalent to a nonsingular monopole on $\mathbf{R}^{3}$, whose moduli space is $\mathbf{R}^{3} \times \mathbf{S}^{1}$. This is clearly false, since the moduli space of a $U(1)$ gauge theory with one charged hypermultiplet is known to be the Taub-NUT space [5, 6], not $\mathbf{R}^{3} \times \mathbf{S}^{1}$. The resolution is that Figure 2(a) corresponds to a monopole with $\ell=1, \ell^{\prime}=0$, just as in 
Figure 2(b), but in a singular gauge. As explained in the Appendix, there is a singular $\theta$-dependent gauge transformation which eliminates the singularity at $\vec{x}=\vec{p}$ but reintroduces one at the monopole core (the singularity is reflected in the "hedgehog" behavior of the Higgs field near the core). In this new gauge the fields are nonsingular at $\vec{x}=\vec{p}$, as expected in a situation like Figure 2(a), with no semi-infinite D3-branes ending on the NS5-branes. Such a "shaggy monopole" has the same moduli space as the normal monopole on the Taub-NUT with $\ell=1, \ell^{\prime}=0$, which in turn is equivalent to a monopole on $\mathbf{R}^{3}$ with Higgs field diverging near the point $\vec{x}=\vec{p}$ as in Eq. (任). The moduli space of the latter is indeed the Taub-NUT space [2].

What happens if one starts with Figure 3(a) and moves the D5-brane inside? According to Ref. [1] the final configuration must be that in Figure 3(b). One might expect a charged hypermultiplet from strings connecting the internal D3-brane with the newly created one. This again would imply a phase transition when the D5-brane crosses the NS5-brane. Moreover, if we move the D5-brane farther to the left, there will be another D3-brane created (see Figure 3(c)) and one might think that two hypermultiplets appear! Is there a phase transition in this case?

The interpretation in terms of monopoles on Taub-NUT space helps us to understand what happens to the moduli spaces and to see that there is no phase transition. Namely, Figure 3(b) corresponds to a "shaggy monopole" with an additional $\ell=-1, \ell^{\prime}=0$ singularity at the center of the Taub-NUT space $\vec{x}=\vec{p}$. The singularities at the monopole core and at $\vec{x}=\vec{p}$ can both be simultaneously eliminated by a gauge transformation (see Appendix), and we are back to the normal monopole with $\ell=0, \ell^{\prime}=0$. The latter is equivalent to a nonsingular $n=1$ monopole on $\mathbf{R}^{3}$, and therefore the moduli space is $\mathbf{R}^{3} \times \mathbf{S}^{1}$, the same as that of the configuration in Figure 3(a). In Figure 3(c) the D3-branes connecting the D5 with NS5's correspond to a Dirac monopole embedded in the subgroup $U(1)_{c m} \subset U(2)=U(1)_{c m} \times S U(2)$, therefore they do not influence the $S U(2)$ monopole at all. (The component of the Higgs field in $U(1)_{\mathrm{cm}}$ corresponds to the center-of-mass motion of the fivebranes.) Thus the moduli space is still $\mathbf{R}^{3} \times \mathbf{S}^{1}$.

Apparently, the naive counting of string modes fails in situations like those in Figures 3(b) and 3(c): in these cases there are no stable fundamental string states connecting the internal D3-branes with the D3-branes stretched between the D5 and NS5-branes.

Similar arguments apply when there is more than one D3 and/or D5brane. 


\section{Moduli Spaces of Singular Monopoles}

There are several approaches to finding metrics on monopole moduli spaces. The most direct one is to use the Nahm transform [10. In principle, this should yield an isometry between the monopole moduli space and the space of solutions of Nahm equations. So far the details have been worked out only for nonsingular $S U(2)$ monopoles [12]. The idea of the minitwistor approach [13, 14] is to encode the monopole data in terms of an algebraic curve in $T \mathbf{P}^{1}$. This curve is then reinterpreted as a spectral curve of Nahm equations, in the spirit of Refs. [15, 14]. This program has been realized for nonsingular monopoles of all classical groups in Ref. [16]. This approach only allows to prove that the moduli spaces of monopoles and Nahm data are diffeomorphic. There is a natural hyperkähler metric on the space of Nahm data, so it is very plausible that these manifolds are in fact isometric. Here we adopt a less rigorous approach, regarding singular $U(2)$ monopoles as a limit of nonsingular $S U(3)$ monopoles. Therefore we can construct the moduli spaces in question by considering a certain limit of Nahm equations for $S U(3)$ monopoles.

Let us recall what Nahm equations for $S U(3)$ monopoles look like according to Ref. [16]. In the case of maximal breaking, which is all we need, $S U(3)$ monopoles are labeled by a pair of nonnegative integers $(n, k)$. For $n<k$ Nahm data consist of two quadruplets

$$
\left(T_{0}^{(\lambda)}(s), T_{1}^{(\lambda)}(s), T_{2}^{(\lambda)}(s), T_{3}^{(\lambda)}(s)\right), \lambda=1,2,
$$

with the first quadruplet defined for $s \in(0,1)$, and the second one defined for $s \in(1, \mu) . T_{i}^{(1)}$ and $T_{i}^{(2)}, i=0, \ldots, 3$ take values in $u(n)$ and $u(k)$, respectively. It is very convenient to combine the functions $T_{i}^{(\lambda)}, i=0, \ldots, 3, \lambda=$ 1,2 , into two quaternions

$$
T^{(\lambda)}=T_{0}^{(\lambda)}+e_{1} T_{1}^{(\lambda)}+e_{2} T_{2}^{(\lambda)}+e_{3} T_{3}^{(\lambda)}, \lambda=1,2,
$$

with $e_{i}$ being the quaternion units. (In what follows we will denote the real $\left(T_{0}\right)$ and imaginary $\left(T-T_{0}\right)$ parts of quaternions by the symbols Re and Im respectively, and think of the purely imaginary quaternions as threecomponent vectors.) Thus one can think of $T^{(1)}$ and $T^{(2)}$ as two functions $T^{(1)}(s):(0,1) \rightarrow u(n) \otimes \mathbf{H}$ and $T^{(2)}(s):(1, \mu) \rightarrow u(k) \otimes \mathbf{H}$. They must satisfy a number of constraints [16]:

(i) Both functions satisfy Nahm equations 


$$
\frac{d T_{i}}{d s}+\left[T_{0}, T_{i}\right]=\frac{1}{2} \varepsilon^{i j k}\left[T_{j}, T_{k}\right], i=1,2,3 .
$$

(ii) $\operatorname{Re} T^{(1)}(s)$ and $\operatorname{Re} T^{(2)}(s)$ extend smoothly to $[0,1]$ and $[1, \mu]$, respectively. $\operatorname{Im} T^{(1)}(s)$ has a simple pole at $s=0$. The residue is an $n$-dimensional irreducible representation of $s u(2)$. $\operatorname{Im} T^{(2)}(s)$ has a simple pole at $s=\mu$ with a residue which is a $k$-dimensional irreducible representation of $s u(2)$.

(iii) $\operatorname{Im} T^{(1)}(s)$ extends smoothly to $(0,1]$. In the neighborhood of $s=1$ $\operatorname{Im} T^{(2)}(s)$ has the following form

$$
T_{i}^{(2)}(s)=\left(\begin{array}{cc}
\rho_{i} / s+O(1) & O\left(s^{(k-n-1) / 2}\right) \\
O\left(s^{(k-n-1) / 2}\right) & T_{i}^{(1)}(1)+O(s)
\end{array}\right), \quad i=1,2,3 .
$$

Here $\rho_{i}=\rho\left(i \sigma_{i} / 2\right), i=1,2,3$, where $\rho$ is a $(k-n) \times(k-n)$ irreducible representation of $s u(2)$ and $\sigma_{i}$ are Pauli matrices.

The set of all Nahm data satisfying conditions (i)-(iii) is invariant with respect to gauge transformations which act in a more-or-less obvious manner: the gauge group is $U(n)$ on the interval $[0,1]$ and $U(k)$ on the interval $[0, \mu]$. To preserve the condition (iii) and the residues of $T^{(1)}, T^{(2)}$ one must also require that at $s=0$ and $s=\mu$ the gauge transformations reduce to identity, and at $s=1$ the "right" gauge group $U(k)$ reduces to the "left" $U(n)$.

For $n>k$ the constraints are the same, with the roles of $T^{(1)}$ and $T^{(2)}$ interchanged. For $n=k$ the Nahm data include, in addition, a quaternionic vector $a \in \mathbf{H}^{n}$, and the condition (iii) is replaced by the following one:

(iii') $\operatorname{Im} T^{(1)}$ and $\operatorname{Im} T^{(2)}$ extend smoothly to $(0,1]$ and $[1, \mu)$, respectively, so that

$$
\operatorname{Im} T_{A B}^{(2)}(1)-\operatorname{Im} T_{A B}^{(1)}(1)=\frac{i}{2} a_{(A} e_{1} \bar{a}_{B)}+\frac{1}{2} a_{[A} \bar{a}_{B]}, \quad A, B=1, \ldots, n .
$$

(The parentheses and brackets denote symmetrization and antisymmetrization, respectively.)

In this case the gauge group $U(n)$ acts also on $a$ from the right, $a_{A} \rightarrow$ $a_{B} g(1)_{B A}$, where $g(s)$ is a gauge transformation.

The space of all Nahm data modulo gauge transformations is diffeomorphic to the space of all $(n, k)$ monopoles [16]. There is a natural hyperkähler metric on the space of Nahm data, and therefore it is expected that the two spaces are isometric. 


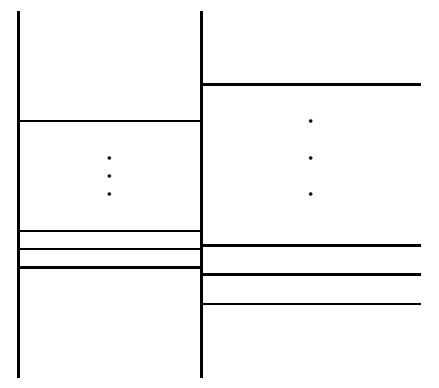

Figure 4: The brane configuration corresponding to singular $U(2)$ monopoles can be obtained as a limit of that corresponding to regular $S U(3)$ monopoles.

To see the metric on the equivalence classes of Nahm data, notice that the gauge group acts triholomorphically on the flat infinite-dimensional hyperkähler manifold consisting of all pairs $\left(T^{(1)}, T^{(2)}\right)$ satisfying (ii) and (iii), except that now the lower right corner of $\operatorname{Im} T^{(2)}(1)$ need not be equal to $\operatorname{Im} T^{(1)}(1)$. (For $n=k$ one must consider instead the space of all triplets $\left(T^{(1)}, T^{(2)}, a\right)$ such that $T^{(1)}$ and $T^{(2)}$ satisfy (ii) and extend smoothly to $s=1$, and $a \in \mathbf{H}^{n}$.) The Nahm equations can be interpreted as moment map equations for gauge transformations which are identity at $s=1$. The boundary conditions for Nahm data at $s=1$ can be interpreted as moment map equations for the action of the residual gauge group, which is the group of all gauge transformations modulo those which are the identity at $s=1$. (This group is $U(\min (n, k))$.) Thus one can use the hyperkähler quotient construction of Hitchin et al. [17 to construct a hyperkähler metric on the space of Nahm data modulo gauge transformations.

To obtain $U(2) n$-monopoles with $k$ singularities of the type $\ell=1, \ell^{\prime}=0$ one should take the limit $\mu \rightarrow \infty$ of $(n, k) S U(3)$ monopoles, fixing the positions of $k$ of them which become infinitely heavy. In this limit $S U(3)$ is broken down to $U(2)$ at a very high scale, so that $(0,1)$ monopoles become point-like Dirac monopoles, while $(1,0)$ monopoles remain smooth. It is easy to see that the magnetic charges carried by Dirac monopoles also come out right. The corresponding brane configuration is shown in Figure 4. From the above description it is clear that in this limit $T^{(2)}$ becomes a function 
defined on $(1,+\infty)$ and satisfying (i) and (iii) (or (iii') if $n=k$ ). A natural boundary condition at $+\infty$ is to require that $\lim _{s \rightarrow+\infty} T^{(2)}(s)$ exist in some gauge, and that $\operatorname{Re} T^{(2)}(+\infty)=0$. Then Nahm equations imply that the matrices $T_{i}^{(2)}(+\infty), i=1,2,3$, commute and can be reduced to a diagonal form $\operatorname{Im} T^{(2)}(+\infty)=\operatorname{diag}\left(\vec{p}_{1}, \ldots, \vec{p}_{k}\right)$ for some $\vec{p}_{\alpha} \in \mathbf{R}^{3}$. It remains to understand what the positions of infinitely heavy monopoles are. The Nahm data just described depend on $k$ vectors $\vec{p}_{\alpha}, \alpha=1, \ldots, k$. It is therefore tempting to identify them as the positions of $k$ infinitely heavy monopoles, i.e. singularities of the Higgs field. This identification can be justified by recalling the physical meaning of Nahm data 18. The variable $s$ is interpreted as the coordinate $x^{6}$ along the horizontal direction in Figure 4 . The matrices $T_{i}^{(2)}$ describe the transverse coordinates of semi-infinite D3-branes. For generic values of $s$ the three matrices $T_{i}^{(2)}$ do not commute, so the notion of the transverse position of a given D3-brane is not defined. However, at $s=+\infty$ the matrices do commute, and their eigenvalues $\vec{p}_{\alpha}$ have the meaning of the D3-branes' asymptotic coordinates in the $\left(x^{3}, x^{4}, x^{5}\right)$ plane. From Section 2 we know that these asymptotic coordinates are precisely the positions of the singularities of the Higgs field.

\section{Examples}

In this section we illustrate the above construction by the examples of $U(1)$ and $S U(2)$ gauge theories with $k$ massive fundamentals. In what follows $\vec{p}_{\alpha}, \alpha=1, \ldots, k$ will be hypermultiplet masses. Let us also recall that $n=1$ (one monopole) corresponds to a $U(1)$ gauge theory, and $n=2$ (two monopoles) corresponds to a $U(2)$ or $S U(2)$ gauge theory.

\section{1 $n=1, k$ arbitrary}

The Nahm data consist of an $\mathbf{H}$-valued function $T^{(1)}(s)$ on $[0,1]$ and a $u(k) \otimes$ $\mathbf{H}$-valued function $T^{(2)}(s)$ on $(1,+\infty) . T^{(2)}$ has a simple pole at $s=1$, and the matching condition (iii) is satisfied. The boundary conditions at $s=+\infty$ are $\operatorname{Re} T^{(2)}(+\infty)=0, \operatorname{Im} T^{(2)}(+\infty)=\operatorname{diag}\left(\vec{p}_{1}, \ldots, \vec{p}_{k}\right)$.

In this case we expect, from field theory, that the moduli space is the $A_{k-1}$ ALF space [5]. To obtain this result in our setup we would have to solve $k \times k$ Nahm equations on a half-line. Alas, we do not know how to do it directly. Fortunately, there is a way to find the moduli space of Nahm equations 
without actually solving them 19. First one splits the three Nahm equations into one complex and one real equation. This amounts to picking a complex structure out of a $\mathbf{P}^{1}$ of available complex structures. The complex equation is invariant under the complexification of the gauge group $G^{\mathbf{C}}$. Donaldson proved that the space of solutions of the complex equation modulo $G^{\mathbf{C}}$ is the same as the space of solutions of all three equations modulo $G$. Thus it suffices to solve the complex equation to determine the moduli space of Nahm equations as a complex manifold. (This is similar to how one computes the moduli space of supersymmetric gauge theories: instead of solving both $D$ and $F$-flatness conditions, one solves only the $F$-flatness conditions modulo the complexification of the gauge group.) Solving the complex equation is easy since it is locally trivial. We refer the reader to Ref. [19] for details, and to Refs. 20, 21, 22 for some applications of this technique. In our case the moduli space turns out to be isomorphic to a hypersurface in $\mathbf{C}^{3}$ specified by the equation

$$
x y=\prod_{\alpha=1}^{k}\left(z-p_{\alpha}\right),
$$

where $p_{\alpha}$ is the "complex" part of $\vec{p}_{\alpha}, p_{\alpha}=p_{\alpha 1}+i p_{\alpha 2}$. This is the complex structure of the resolution of $A_{k-1}$ singularity.

To find the metric one needs to know all three complex structures, however. To this end one has to vary the arbitrary complex structure we picked in the beginning and see how variables in Eq. (11) change 22. The result is rather simple to describe: $p_{\alpha}$ are fixed real sections of $\mathcal{O}(2), z$ is the coordinate in the fiber of the line bundle $T \mathbf{P}^{1}=\mathcal{O}(2)$ over the $\mathbf{P}^{1}$ of complex structures, while $x$ and $y$ take values in the line bundles $L(k)$ and $L^{-1}(k)$ over $T \mathbf{P}^{1}$, respectively. (In the notation of Ref. [14] $L^{x}(k)$ is a line bundle over $T \mathbf{P}^{1}$ with a transition function $\zeta^{-k} e^{x \eta / \zeta}$, where $\zeta$ is the coordinate on $\mathbf{P}^{1}$ and $\eta$ is the coordinate in the tangent space at $\zeta, d \zeta(\eta)=1$.) With such identification Eq. (11) describes the twistor space of the $A_{k-1}$ ALF space [23].

\section{$6.2 n=2, k=1$}

The Nahm data consist of a $u(2) \otimes \mathbf{H}$-valued function $T^{(1)}(s)$ on $(0,1]$ and a $u(1) \otimes \mathbf{H}$-valued function $T^{(2)}(s)$ on $[1,+\infty) . T^{(1)}$ has a simple pole at $s=0$ with residue $e_{1} \rho_{1}+e_{2} \rho_{2}+e_{3} \rho_{3}$, where $\rho_{i}=i \sigma_{i} / 2, i=1,2,3$. The boundary conditions at $s=+\infty$ are $\operatorname{Re} T^{(2)}(+\infty)=0, \operatorname{Im} T^{(2)}(+\infty)=\vec{p}$. At $s=1$ the matching condition (iii) is satisfied. 
It proves convenient to perform the hyperkähler quotient in two steps. First we take the quotient with respect to gauge transformations which are the identity at $s=1$. This amounts to solving Nahm equations on two intervals separately and finding their moduli spaces of solutions. For $s \in(1,+\infty)$ Nahm equations just tell us that $\operatorname{Im} T^{(2)}$ is independent of $s$ and equal to $\vec{p}$, i.e. the moduli space is just a point. Solving Nahm equations for $2 \times 2$ matrices on $(0,1)$ is also elementary, since the equations can be reduced to those of the Euler top. In fact, for $s \in(0,1]$ the moduli space of solutions with boundary behavior as described above has been investigated by Dancer [21]. It turns out that the moduli space is a 12-dimensional hyperkähler manifold $M^{12}$ of the form $\mathbf{R}^{3} \times \mathbf{S}^{1} \times M^{8}$, where $M^{8}$ is also hyperkähler and irreducible. $M^{8}$ admits a triholomorphic action of $S U(2)$.

Second, we take the quotient with respect to the $U(1)$ group "living" at $s=1$. This $U(1)$ is a subgroup of $U(2)$ which is the group of all gauge transformations modulo those which reduce to identity at $s=1$. More concretely, the $U(1)$ acts on $\mathbf{R}^{3} \times \mathbf{S}^{1} \times M^{8}$ as follows: it rotates the $\mathbf{S}^{1}$, and it acts on $M^{8}$ as a maximal torus of the triholomorphic $S U(2)$ mentioned in the end of the previous paragraph. The boundary condition (iii) implies that the level of the quotient is $2 \vec{p}$. The net result is an 8-dimensional hyperkähler manifold depending on $\vec{p}$ as a parameter. It is the moduli space of two monopoles with a fixed singularity at $\vec{x}=\vec{p}$ and corresponds to the $U(2)$ gauge theory with one massive fundamental hypermultiplet.

If one wishes to obtain the Coulomb branch of the $S U(2)$ theory with the same matter content, one should perform a further $U(1)$ hyperkähler quotient (i.e. pass to the centered monopole moduli space). This $U(1)$ is easily identified: it acts on $\mathbf{R}^{3} \times \mathbf{S}^{1}$ by rotating the $\mathbf{S}^{1}$. The level of the quotient is simply the position of the monopoles' center of mass. It can always be absorbed into $\vec{p}$, so we can set it to zero. Performing this $U(1)$ quotient rids the $M^{12}$ of the $\mathbf{R}^{3} \times \mathbf{S}^{1}$ factor. Thus we conclude that the moduli space of the $S U(2)$ gauge theory with one fundamental is the hyperkähler quotient of $M^{8}$ by $U(1)$ at level $2 \vec{p}$. This is exactly the four-dimensional manifold constructed by Dancer in Ref. [21] and proposed in Ref. [6] as a candidate for the Coulomb branch. Moreover, we showed above that $\vec{p}$ should be identified as the mass of the hypermultiplet. This agrees with Ref. [6] where it was suggested that the level of the quotient should be twice the mass of the fundamental. 


\section{$6.3 n=2, k=2$.}

In this case the Nahm data consist of a $u(2) \otimes \mathbf{H}$-valued function $T^{(1)}(s)$ on $(0,1]$, a $u(2) \otimes \mathbf{H}$-valued function $T^{(2)}(s)$ on $[1,+\infty)$, and a quaternionic vector $a \in \mathbf{H}^{2}$. Both functions satisfy Nahm equations. $T^{(1)}$ has a simple pole at $s=0$ with residue $e_{1} \rho_{1}+e_{2} \rho_{2}+e_{3} \rho_{3}$, where $\rho_{i}=i \sigma_{i} / 2, i=1,2,3$. The boundary conditions at $s=+\infty$ are $\operatorname{Re} T^{(2)}(+\infty)=0, \operatorname{Im} T^{(2)}(+\infty)=$ $\operatorname{diag}\left(\vec{p}_{1}, \vec{p}_{2}\right)$. At $s=1$ the matching condition (iii') is satisfied.

Again we split the calculation in two steps. The solution of Nahm equations for $s \in(0,1)$ is the same as before. To solve the equations on $(1,+\infty)$, we split $T^{(2)}$ into a part proportional to the identity matrix and a traceless matrix. The equations for the "identity" part simply say that $\operatorname{Tr}\left(\operatorname{Im} T^{(2)}\right)$ is independent of $s$ and equal to $\vec{p}_{1}+\vec{p}_{2}$. The equations for the traceless part can be solved in terms of hyperbolic functions. After one performs the quotient with respect to the $U(2)$ gauge group which degenerates to the identity at $s=1$ and to $U(1) \times U(1)$ at $s=+\infty$, one gets a four-dimensional moduli space $M_{E H}$. Its metric can be computed to be the two-center GibbonsHawking (or Eguchi-Hanson) metric with $\left|\vec{p}_{1}-\vec{p}_{2}\right|$ being the distance between the centers. Actually, this is a particular case of Kronheimer's construction of hyperkähler metrics on the coadjoint orbits of a complex group $G$ [20]. Kronheimer's construction also uses Nahm equations, and for $G=S L(2, \mathbf{C})$ coincides with ours. (The coadjoint orbit here happens to be isomorphic to $T \mathbf{P}^{1}$ as a complex manifold.) The Eguchi-Hanson metric admits a triholomorphic action of $S U(2)$.

The second step is to take the hyperkähler quotient of

$$
M^{12} \times \mathbf{H}^{2} \times M_{E H}
$$

with respect to $U(2)$. This residual $U(2)$ is the quotient of all gauge transformations by those which reduce to the identity at $s=1$. The subgroup $U(1) \subset U(2)=U(1) \times S U(2)$ acts on $M^{12}=\mathbf{R}^{3} \times \mathbf{S}^{1} \times M^{8}$ by rotating $\mathbf{S}^{1}$, and on $a \in \mathbf{H}^{2}$ by right multiplication by $\exp \left(e_{1} \phi\right)$. The matching condition (iii') means that the $U(1)$ quotient should be performed at level $\vec{p}_{1}+\vec{p}_{2}$. The $S U(2)$ subgroup acts on the $M^{8}$ part of $M^{12}$ and on the Eguchi-Hanson space $M_{E H}$. It also acts on $a$ by the right multiplication $a \rightarrow a g^{t}$.

The quotient manifold is an 8-dimensional hyperkähler manifold depending on $\vec{p}_{1}$ and $\vec{p}_{2}$ as parameters. It is the moduli space of two monopoles with two fixed singularities at $\vec{p}_{1}$ and $\vec{p}_{2}$ and corresponds to the $U(2)$ gauge theory with two massive fundamental hypermultiplets. 
To obtain the Coulomb branch of the $S U(2)$ theory we must "center" the monopoles, as in the previous example. The position of the center of mass can be set to zero without loss of generality. As earlier, "centering" monopoles is achieved by taking a $U(1)$ quotient. This procedure eliminates the $\mathbf{R}^{3} \times \mathbf{S}^{1}$ factor of $M^{12}$. Then we need to compute the hyperkähler quotient of

$$
M^{8} \times \mathbf{H}^{2} \times M_{E H}
$$

by $U(2)=U(1) \times S U(2)$, where $U(1)$ acts only on $\mathbf{H}^{2}$ by right multiplication, and $S U(2)$ acts on all three factors. The level of the quotient is $\vec{p}_{1}+\vec{p}_{2}$. Since $U(1)$ acts so simply, we can perform the quotient with respect to it explicitly (see e.g. Ref [24]). The final result is that the the Coulomb branch of the $S U(2)$ theory with two fundamentals is the hyperkähler quotient of $M^{8} \times M_{E H}^{\prime} \times M_{E H}$ with respect to $S U(2)$. Both $M_{E H}^{\prime}$ and $M_{E H}$ are the twocenter Gibbons-Hawking (Eguchi-Hanson) spaces with distances between the centers $\vec{p}_{1}+\vec{p}_{2}$ and $\vec{p}_{1}-\vec{p}_{2}$ respectively.

\section{$6.4 n=2, k=3$}

It is convenient to slightly change our point of view and regard two $U(2)$ monopoles with three singularities as a limit of $S U(4)(1,2,2)$ monopoles, rather than the limit of $S U(3)(2,3)$ monopoles. The limit is such that $(1,$, and $(,, 2)$ monopoles become infinitely heavy. The corresponding brane construction is shown in Figure 5. The Nahm data consist of three functions $T^{(0)}:(-\infty, 0] \rightarrow u(1) \otimes \mathbf{H}, T^{(1)}:[0,1] \rightarrow u(2) \otimes \mathbf{H}, T^{(2)}:[1,+\infty) \rightarrow u(2) \otimes \mathbf{H}$ and a quaternionic vector $a \in \mathbf{H}^{2}$. The boundary conditions at $s= \pm \infty$ are $\operatorname{Re} T^{(0)}(-\infty)=\operatorname{Re} T^{(2)}(+\infty)=0, \operatorname{Im} T^{(0)}(-\infty)=\vec{p}_{1}, \operatorname{Im} T^{(2)}(+\infty)=$ $\operatorname{diag}\left(\vec{p}_{2}, \vec{p}_{3}\right)$. Here $\vec{p}_{\alpha}$ are the positions of the singularities of the Higgs field. The matching condition at $s=0$ is

$$
\operatorname{Im} T^{(1)}(0)_{2,2}=\operatorname{Im} T^{(0)}(0) .
$$

The matching condition at $s=1$ is

$$
\operatorname{Im} T_{A B}^{(2)}(1)-\operatorname{Im} T_{A B}^{(1)}(1)=\frac{i}{2} a_{(A} e_{1} \bar{a}_{B)}+\frac{1}{2} a_{[A} \bar{a}_{B]}, A, B=1,2 .
$$

The advantage of this point of view is that we already know what the moduli spaces of solutions of Nahm equations look like for $s \in(-\infty, 0)$ and $s \in(1,+\infty)$ : in the first instance it is a point, and in the second instance it 


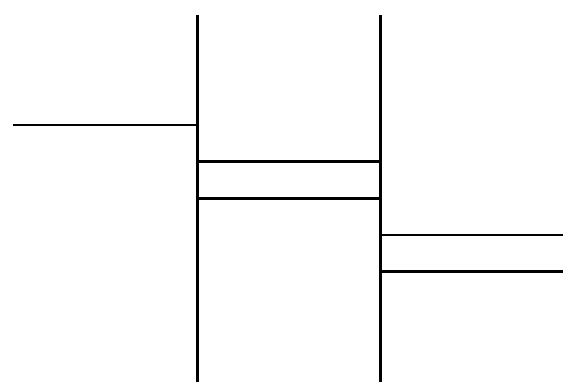

Figure 5: A $U(2)$ 2-monopole with three singularities is a limit of a regular $(1,2,2) S U(4)$ monopole.

is a Eguchi-Hanson space $M_{E H}$ with distance between the centers $\left|\vec{p}_{2}-\vec{p}_{3}\right|$. For $s \in(0,1)$ we now have to analyze the space of solutions of $2 \times 2 \mathrm{Nahm}$ equations with nonsingular boundary behavior. Luckily, this has also been done by Dancer [ 8$]$. The moduli space is a 16-dimensional hyperkähler manifold $N^{16}$ which has the form $\mathbf{R}^{3} \times \mathbf{S}^{1} \times N^{12}$. $N^{12}$ is hyperkähler and irreducible. It admits a triholomorphic $S U(2)_{L} \times S U(2)_{R}$ action. (We call these two $S U(2)$ 's $S U(2)_{L}$ and $S U(2)_{R}$ because they originate from the action of the residual gauge group at $s=0$ and $s=1$.)

If we perform the hyperkähler quotient in two steps, as before, on the first step we get $N^{16} \times \mathbf{H}^{2} \times M_{E H}$. On the second step we take the hyperkähler quotient with respect to $U(1) \times U(2)$. $U(1)$ acts on the $N^{12}$ part of $N^{16}$ by a maximal torus of $S U(2)_{L}$, and the level of this quotient is $2 \vec{p}_{1}$. $U(2)=U(1) \times S U(2)$ acts as follows: its $U(1)$ subgroup acts only on $\mathbf{H}^{2}$ by right multiplication by $\exp \left(e_{1} \phi\right)$, while its $S U(2)$ subgroup acts on all three factors, the action on $N^{16}$ being that of $S U(2)_{R}$. The resulting manifold is the Coulomb branch of the $U(2)$ gauge theory with three hypermultiplets. "Centering" the monopole moduli space we get the following description of the Coulomb branch of the $S U(2)$ theory with three hypermultiplets: it is a hyperkähler quotient of

$$
N^{12} \times M_{E H}^{\prime} \times M_{E H}
$$

with respect to $U(1) \times S U(2)$, where $M_{E H}^{\prime}$ and $M_{E H}$ are Eguchi-Hanson spaces with distances between the centers $\left|\vec{p}_{2}+\vec{p}_{3}\right|$ and $\left|\vec{p}_{2}-\vec{p}_{3}\right|$, respectively. Here $U(1)$ acts only on $N^{12}$ by a maximal torus of $S U(2)_{L}$, and the level is 


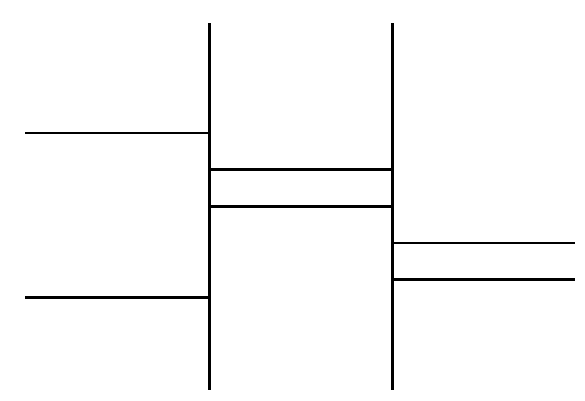

Figure 6: A $U(2)$ 2-monopole with four singularities is a limit of a regular $(2,2,2) S U(4)$ monopole.

$2 \vec{p}_{1}$. $S U(2)$ acts on all three factors, the action on $N^{12}$ being that of $S U(2)_{R}$.

\section{$6.5 n=2, k=4$}

Similarly to the previous example, we regard two monopoles with four singularities as a limit of $S U(4)(2,2,2)$ monopoles. The limit is such that $(2,$, and $(,, 2)$ monopoles become infinitely heavy. The corresponding brane configuration is shown in Figure 6.

We do not spell out in detail the manipulations with hyperkähler quotients, since they are almost the same as in the previous example. We just give the result for the Coulomb branch of the $S U(2)$ gauge theory with four fundamental hypermultiplets: it is a hyperkähler quotient of

$$
M_{E H} \times M_{E H}^{\prime} \times N^{12} \times M_{E H}^{\prime \prime} \times M_{E H}^{\prime \prime \prime}
$$

with respect to $S U(2)_{L} \times S U(2)_{R}$, where, as the names suggest, $S U(2)_{L}$ acts on $M_{E H}, M_{E H}^{\prime}, N^{12}$, and $S U(2)_{R}$ acts on $N^{12}, M_{E H}^{\prime \prime}, M_{E H}^{\prime \prime \prime}$. The spaces $M_{E H}, M_{E H}^{\prime}, M_{E H}^{\prime \prime}$, and $M_{E H}^{\prime \prime \prime}$ are Eguchi-Hanson spaces with distances between the centers $\left|\vec{p}_{1}+\vec{p}_{2}\right|,\left|\vec{p}_{1}-\vec{p}_{2}\right|,\left|\vec{p}_{3}+\vec{p}_{4}\right|$, and $\left|\vec{p}_{3}-\vec{p}_{4}\right|$. Arguments from M-theory [5] or field theory [6] show that this space is an ALF gravitational instanton of type $D_{4}$. Thus we have a rather simple construction of such a space. 


\section{Complex Structures on Moduli Spaces}

Our description of the metrics on the Coulomb branches is implicit. This is hardly a drawback, since an explicit formula would be horribly complicated (see e.g. Ref. [21] where the metric corresponding to $n=2, k=1$ is discussed). The only exception is the case of $U(1)$ gauge theories where the moduli space is of the multi-Taub-NUT form. For general $n$ it is possible to give an explicit description of the Coulomb branches as complex manifolds. We remind that hyperkähler manifolds have three different complex structures $I, J, K$, but we will concentrate on just one of them, say $I$. We will limit the discussion of the complex structures to the cases studied in the previous section.

Computing the complex structures allows us to perform some checks of the metrics. It follows both from M-theory [5] and field theory [6] considerations that the Coulomb branch of $S U(2)$ gauge theory with $k$ fundamentals is an ALF gravitational instanton of type $D_{k}$. We will be able to see that indeed our manifolds are resolutions of $D_{k}$ singularities, at least for $k \leq 4$. A more detailed check can be performed by comparison with the SeibergWitten solutions of the corresponding $N=2 S U(2)$ gauge theories in four dimensions. Recall that in four dimensions the description of the Coulomb branch of an $N=2 S U(2)$ gauge theory involves a complex torus fibered over a complex plane [25]. The plane is the moduli space of the theory, while the torus is an auxiliary object whose $\tau$-parameter is the low-energy gauge coupling. Upon compactification to three dimensions the total space of this fibration becomes the moduli space of the corresponding three-dimensional $N=4$ theory [6]. Moreover, it was argued [6] that the complex structure remains the same as in four dimensions (of course, after compactification the moduli space grows another two complex structures which we disregard in this section.) We will see this quite explicitly below.

To compute the complex structure on the moduli space of Nahm equations we followed the approach of Donaldson [19] (see Section 6.1). The calculation, although simple in principle, is rather cumbersome and will be presented elsewhere 26]. 


\section{1 $S U(2)$ theory with one hypermultiplet}

The complex structure is given by

$$
y^{2}=x^{2} z+2 p x+1
$$

where $p$ is the "complex" part of the hypermultiplet mass parameter $\vec{p}$. The Seiberg-Witten solution is

$$
y^{2}=x^{2}(x-u)+2 m x+1,
$$

where $m$ is the hypermultiplet mass in four dimensions. Obviously, the two complex structures agree after on sets $z=x-u, p=m$ in Eq. (18).

\section{2 $S U(2)$ theory with two hypermultiplets}

The complex structure is given by

$$
y^{2}=x^{2} z-z+2 x p_{1} p_{2}-\left(p_{1}^{2}+p_{2}^{2}\right) .
$$

The Seiberg-Witten solution is

$$
y^{2}=\left(x^{2}-1\right)(x-u)+2 x m_{1} m_{2}-\left(m_{1}^{2}+m_{2}^{2}\right) .
$$

Eq. (20) agrees with Eq. (21) if one sets $z=x-u, p_{1}=m_{1}, p_{2}=m_{2}$.

\section{3 $S U(2)$ theory with three hypermultiplets}

The complex structure is given by

$$
y^{2}=x^{2} z-z^{2}-z\left(p_{1}^{2}+p_{2}^{2}+p_{3}^{2}\right)+2 x p_{1} p_{2} p_{3}-\left(p_{1}^{2} p_{2}^{2}+p_{1}^{2} p_{3}^{2}+p_{2}^{2} p_{3}^{2}\right) .
$$

The Seiberg-Witten solution is

$$
\begin{aligned}
y^{2}= & x^{2}(x-u)-(x-u)^{2}-(x-u)\left(m_{1}^{2}+m_{2}^{2}+m_{3}^{2}\right)+2 x m_{1} m_{2} m_{3} \\
& -\left(m_{1}^{2} m_{2}^{2}+m_{2}^{2} m_{3}^{2}+m_{1}^{2} m_{3}^{2}\right) .
\end{aligned}
$$

Eqs. (22) and (23) agree if one sets $z=x-u, p_{1}=m_{1}, p_{2}=m_{2}, p_{3}=m_{3}$. 


\section{4 $S U(2)$ theory with four hypermultiplets}

The complex structure is given by

$$
\begin{aligned}
y^{2}= & x^{2} z-z^{3}+z^{2}\left(S_{2}-\frac{S_{1}^{2}}{2}\right)-z\left(\frac{3 S_{1}^{4}}{32}-\frac{3 S_{1}^{2} S_{2}}{8}+\frac{S_{2}^{2}+S_{1} S_{3}}{4}+\frac{S_{4}}{2}\right) \\
& +x\left(\frac{S_{1}^{4}}{32}-\frac{S_{1}^{2} S_{2}}{8}+\frac{S_{1} S_{3}}{4}-\frac{S_{4}}{2}\right)-\frac{S_{1}^{6}}{128}+\frac{3 S_{1}^{4} S_{2}}{64}-\frac{S_{1}^{2} S_{2}^{2}+S_{1}^{3} S_{3}}{16} \\
& +\frac{S_{1} S_{2} S_{3}-S_{3}^{2}}{8}+\frac{S_{2} S_{4}}{4},
\end{aligned}
$$

where $S_{\ell}$ is an elementary symmetric polynomial in $p_{1}, \ldots, p_{4}$ of degree $\ell$, i.e. $S_{1}=p_{1}+p_{2}+p_{3}+p_{4}, \ldots, S_{4}=p_{1} p_{2} p_{3} p_{4}$. One immediately sees that this is indeed a resolution of $D_{4}$ singularity. To compare with the Seiberg-Witten solution in $D=4$ it is best to think of $S U(2)$ theory with four flavors as a special case of $S p(2 n)$ theory with $2 n+2$ flavors. Specializing the formula for the $\operatorname{Sp}(2 n)$ curve given in Ref. [27] to $n=1$ we get the following hyperelliptic curve:

$$
x y^{2}=\left(x(x-u)+g \prod_{l=1}^{4} m_{l}\right)^{2}-g^{2} \prod_{l=1}^{4}\left(x-m_{l}^{2}\right) .
$$

Eqs. (24) and (25) agree if in Eq. (25) one sets $m_{l}=i p_{l} / \sqrt{2 g}, l=1, \ldots, 4$, and makes the following substitution:

$$
\begin{aligned}
& y \rightarrow \frac{y}{\sqrt{g}}, \\
& x \rightarrow \frac{1}{2 g}\left(x-z+\frac{S_{2}}{2}-\frac{S_{1}^{2}}{4}\right), \\
& u \rightarrow \frac{1}{2}\left(x+3 z-\frac{S_{2}}{2}+\frac{S_{1}^{2}}{4}\right)+\frac{1}{2 g}\left(x-z+\frac{S_{2}}{2}-\frac{S_{1}^{2}}{4}\right) .
\end{aligned}
$$

\section{Summary and Conclusions}

In this paper we showed that the Coulomb branches of $D=3, N=4 S U(n)$ and $U(n)$ gauge theories with $k$ fundamental hypermultiplets are identical to the (centered) moduli spaces of $n U(2)$ monopoles with $k$ singularities. We then constructed the latter spaces as infinite dimensional hyperkähler

quotients (i.e. as the moduli spaces of Nahm equations). This amounts to 
an implicit description of the exact metrics on the Coulomb branches. For the simplest cases of $U(1)$ and $S U(2)$ gauge theories we also computed the complex structures on the moduli spaces and compared with expectations from field theory. This provides a check of the correspondence between the monopole moduli spaces and the Coulomb branches. An even more stringent check is afforded by the comparison with Seiberg-Witten solutions of $D=4$, $N=2$ theories which upon dimensional reduction yield our $D=3, N=$ 4 theories. It was argued in Ref. [6] that that a "distinguished" complex structure of the $D=3$ moduli space is the same as the complex structure of the Seiberg-Witten fibration in $D=4$. We checked that this is indeed the case for $S U(2)$ theory with up to four fundamental hypermultiplets.

As a by-product, we constructed ALF gravitational instantons of type $D_{k}$ for any positive $k$ as centered moduli spaces of two monopoles with $k$ singularities. A detailed discussion of their metrics and twistor spaces will be presented in forthcoming papers [3, 26]. It remains to be seen if these methods can be exploited to construct $E_{k}$ gravitational instantons.

Finally, using the monopole interpretation, we argued for the absence of phase transitions in the gauge theory whenever a D5-brane crosses any of the NS5-branes. It turns out that brane creation is not sufficient to explain this: in addition one has to postulate that in some brane configurations described in Section 1 certain string modes are absent, contrary to naive expectations.

\section{Acknowledgements}

We would like to thank Amihay Hanany, Nigel Hitchin, John H. Schwarz, and Edward Witten for helpful conversations.

\section{Appendix: "Shaggy Monopoles"}

In order to define "shaggy monopoles" and give their interpretation in terms of D-branes, first let us look at self-dual $U(2)$ connections on $\mathbf{R}^{3} \times \mathbf{S}^{1}$. We can think of these connections as living on the world-volume of two coincident D6-branes wrapped around $\mathbf{R}^{3} \times \mathbf{S}^{1}$. Let $R_{A}$ be the radius of $\mathbf{S}^{1}$, and let $\theta$ be the coordinate along it. We consider the connections with fixed second Chern class and fixed conjugacy class of holonomy around $\mathbf{S}^{1}$ at infinity. If the asymptotic eigenvalues of $A_{0}$ (the $\theta$-component of the gauge field) are $\mu_{1}$ 
and $\mu_{2}$, the holonomy at infinity is conjugate to

$$
W=\left(\begin{array}{cc}
e^{2 \pi i R_{A} \mu_{1}} & 0 \\
0 & e^{2 \pi i R_{A} \mu_{2}}
\end{array}\right) .
$$

Let's assume that $\mu_{1}>\mu_{2}$, for definiteness. After one T-dualizes along the $\theta$ direction $2 \pi \mu_{1}$ and $2 \pi \mu_{2}$ are interpreted as the $x^{6}$ positions of the D5branes (we set the string scale $\alpha^{\prime}$ to 1 ). Now with two D5-branes at points $x^{6}=2 \pi \mu_{1}$ and $x^{6}=2 \pi \mu_{2}$ there are two ways to stretch a D3-brane between them. After T-duality in the $x^{6}$ both types of stretched D3-branes turn into $U(2)$ monopoles on $\mathbf{R}^{3} \times \mathbf{S}^{1}$, but with different asymptotic eigenvalues of $A_{0}$. Namely, the eigenvalues are $\mu_{1}, \mu_{2}$ in one case and $\mu_{2}+1 / R_{A}, \mu_{1}$ in the other [11]. Both configurations are in fact t'Hooft-Polyakov monopoles, with $A_{0}$ playing the role of the Higgs field, consequently they are $\theta$-independent.

Of course the eigenvalues of $A_{0}$ can be changed by a $\theta$-dependent gauge transformation. For example, to change the eigenvalues from $\mu_{2}+1 / R_{A}, \mu_{1}$ to $\mu_{1}, \mu_{2}$ one has to use the following gauge transformation:

$$
g=\exp \left(\frac{i \theta}{2 R_{A}}\left[\frac{A_{0}-\frac{1}{2} \operatorname{tr} A_{0}}{\left\|A_{0}-\frac{1}{2} \operatorname{tr} A_{0}\right\|}-1\right]\right),
$$

where we defined $\|\phi\|^{2}=\frac{1}{2} \operatorname{tr} \phi^{2}$. Simultaneously this transformation makes $A_{i} \theta$-dependent and singular at every point $\vec{q}$ where the traceless part of $A_{0}$ vanishes. One can think of such a point as the center of the monopole (there is just one such point for a single smooth t'Hooft-Polyakov monopole). The gauge transformation Eq. (28) creates a "hedgehog" at the monopole center, in the sense that the eigenvalues of the Higgs field approach $1 / R_{A}, 0$ as $\vec{x} \rightarrow \vec{q}$, but the direction of the Higgs field in the $u(2)$ algebra depends on the direction of approach. This singular and $\theta$-dependent $U(2)$ connection on $\mathbf{R}^{3} \times \mathbf{S}^{1}$ is what we call a "shaggy monopole." Naturally, the moduli space of a "shaggy monopole" is the same as the moduli space of a regular monopole from which it was obtained by a gauge transformation.

We would like to stress that if only D3-branes of one type are present, one can always use the gauge in which the corresponding gauge configuration is $\theta$-independent. But if D3-branes stretched both ways are present, then $\theta$ dependence cannot be removed by a gauge transformation. In particular the "Wilson-line instanton" of Ref. [11] is $\theta$-dependent.

Now we consider a monopole of magnetic charge 1 on the $A_{k-1}$ ALF space. Let the asymptotic radius of the compact direction be $R_{A}$. As in the case of 
$\mathbf{R}^{3} \times \mathbf{S}^{1}$ we want to fix the holonomy at infinity. Monopoles on the $A_{k-1}$ ALF space can be obtained from smooth BPS monopoles on $\mathbf{R}^{3}$ with asymptotic eigenvalues of the Higgs field at infinity either $\mu_{1}, \mu_{2}$ or $\mu_{2}+1 / R_{A}, \mu_{1}$, the holonomy being the same. Let us choose the latter possibility. Performing the change of variables Eq. (3) we get a $\theta$-independent connection $\tilde{A}$ on the $A_{k-1}$ ALF space. Since $V^{-1}\left(\vec{p}_{\alpha}\right)=0$ for all $\alpha$, the traceless part of $\tilde{A}_{0}$ vanishes not only at the monopole center, but also at the $k$ centers of the $A_{k-1}$ ALF space. Now suppose we want to change the asymptotic eigenvalues of $\tilde{A}_{0}$ to $\mu_{1}, \mu_{2}$. To this end we perform the gauge transformation as in Eq. (28). The new connection will be singular at the monopole center, as well as at $\vec{x}=\vec{p}_{\alpha}, \alpha=1, \ldots, k$. Thus a smooth monopole on the $A_{k-1}$ ALF space with all $\ell_{\alpha}$ equal to zero is gauge-equivalent to a "shaggy monopole" which has singularities at the monopole core and at the centers of the $A_{k-1}$ ALF space.

On the other hand we can start with a $\theta$-independent monopole on the $A_{k-1}$ ALF space which has $\ell_{\alpha}=1, \ell_{\alpha}^{\prime}=0, \alpha=1, \ldots, k$. After the gauge transformation inverse to that in Eq. (28) it turns into a connection which has $\hat{A}_{0}\left(\vec{p}_{\alpha}\right)=0, \alpha=1, \ldots, k$, and a "hedgehog" in the monopole center. (Notice that in Section 3 we set $R_{A}=2$.) Thus we can trade the singularities at

the centers of the $A_{k-1}$ ALF space for a similar singularity at the monopole center by means of a singular gauge transformation.

\section{References}

[1] A. Hanany and E. Witten, "Type IIB Superstrings, BPS Monopoles, and Three-Dimensional Gauge Dynamics," Nucl. Phys. B492 152-190 (1997), hep-th/9611230.

[2] P. B. Kronheimer, "Monopoles and Taub-NUT Metrics," M. Sc. Thesis, Oxford, 1985.

[3] S. A. Cherkis and A. Kapustin, "Singular Monopoles and Gravitational Instantons," in preparation.

[4] K. Intriligator and N. Seiberg, "Mirror Symmetry in Three Dimensional Gauge Theories," Phys. Lett. B387 513 (1996), hep-th/9607207.

[5] N. Seiberg, "IR Dynamics on Branes and Space-Time Geometry," Phys. Lett. B384 81-85 (1996), hep-th/9606017. 
[6] N. Seiberg, E. Witten, "Gauge Dynamics and Compactification to Three Dimensions," hep-th/9607163.

[7] P. B. Kronheimer, "The Construction of ALE spaces as Hyper-Kähler Quotients," J. Diff. Geom. 29 665-683 (1989).

[8] A. S. Dancer, "Dihedral Singularities and Gravitational Instantons," J. Geom. Phys. 12 77-91 (1993).

[9] M. R. Douglas, "Branes within Branes," hep-th/9512077.

[10] W. Nahm, "Self-dual Monopoles and Calorons," Lecture Notes in Physics 201, Springer, New York, 1984.

[11] K. Lee and P. Yi, "Monopoles and Instantons on Partially Compactified D-branes," Phys. Rev. D56 3711-3717 (1997), hep-th/9702107.

[12] H. Nakajima, "Monopoles and Nahm Equations," in Sanda 1990, Proceedings, Einstein metrics and Yang-Mills connections.

[13] N.J. Hitchin, "Monopoles and Geodesics," Comm. Math. Phys. 83 579602 (1982).

[14] N.J. Hitchin, "On the Construction of Monopoles," Comm. Math. Phys. 89 145-190 (1983).

[15] M. Adler and P. van Moerbeke, "Linearization of Hamiltonian Systems, Jacobi Varieties, and Representation Theory," Adv. Math. 38 318-379 (1980).

[16] J. Hurtubise and M. K. Murray, "On the Construction of Monopoles for the Classical Groups," Comm. Math. Phys. 122 35-89 (1989).

[17] N. J. Hitchin, A. Karlhede, U. Lindström, and M. Roček, "Hyperkähler Metrics and Supersymmetry," Comm. Math. Phys. 108 535-589 (1987).

[18] D.-E. Diaconescu, "D-branes, Monopoles, and Nahm Equations," hepth/9608163.

[19] S. K. Donaldson, "Nahm's Equations and the Classification of Monopoles," Comm. Math. Phys. 96 387-407 (1984). 
[20] P. B. Kronheimer, "A Hyper-Kählerian Structure on Coadjoint Orbits of a Semisimple Complex Group," J. Lond. Math. Soc. 42, part 2, 193-208 (1990).

[21] A. S. Dancer, "Nahm Equations and Hyperkähler Geometry", Comm. Math. Phys. 158 545-568 (1993).

[22] A. S. Dancer, "A Family of Hyperkähler Manifolds," Quart. J. Math. Oxford (2), 45 463-478 (1994).

[23] N. J. Hitchin, "Twistor Construction of Einstein Metrics," in Durham 1983, Global Riemannian Geometry.

[24] G. W. Gibbons and P. Rychenkova, "Hyperkähler Quotient Construction of BPS Monopoles' Moduli Spaces," hep-th/9608085.

[25] N. Seiberg and E. Witten, "Electric-Magnetic Duality, Monopole Condensation, and Confinement in $N=2$ Supersymmetric Yang-Mills Theory," Nucl. Phys. B426 19 (1994), hep-th/9407087; "Monopoles, Duality, and Chiral Symmetry Breaking in $N=2$ Supersymmetric QCD," Nucl. Phys. B431 484 (1994), hep-th/9408099.

[26] S. A. Cherkis and A. Kapustin, " $D_{k}$ Gravitational Instantons and Nahm Equations," in preparation.

[27] P. C. Argyres and A. D. Shapere, "The Vacuum Structure of N=2 SuperQCD with Classical Gauge Groups," Nucl. Phys. B461 437-459 (1996), hep-th/9509175. 